\title{
Problematizar y deconstruir el concepto hegemónico de Política Exterior Feminista desde Abya Yala: hacia una propuesta de gradualidad en la implementación
}

\section{Juan Martín Barbas, Dulce Daniela Chaves y Mariel Renée LuCERO*}

\section{RESUMEN}

Desde finales del 2014, tras el anuncio de Suecia de embarcarse en una Política Exterior Feminista (PEF), dicho concepto ha sido utilizado de forma amplia en ámbitos académicos y políticos, sin que se haya avanzado en la deconstrucción y delimitación del mismo, desde una mirada plural y crítica del feminismo. Las formulaciones teóricas existentes, provenientes casi todas de académicas/os estadounidenses y/o europeas/os, se limitan a registrar una serie de características mínimas y ambiguas, influenciadas por una lectura liberal y etnocéntrica de los feminismos. Esto permite que se utilice la noción de PEF para nombrar situaciones diversas, y que se establezca, al menos desde lo simbólico, un signo de igualdad entre casos que poco tienen en común; como pueden ser las políticas exteriores de Suecia, Canadá y México. Esta situación no resulta neutral, ya que, por un lado, le quita trascendencia y contundencia a las propuestas que desde hace décadas los feminismos vienen elaborando en materia de teoría de las Relaciones Internacionales; y por el otro, le permite a funcionarios/as, intelectuales y comunicadores/as, apropiarse y hacer un uso superficial de postulados feministas forjados al calor de las intensas luchas que las mujeres, y otros sectores feminizados, vienen llevando a cabo históricamente.

Frente a este panorama, resulta necesaria una disputa por el sentido que recupere los elementos más trasformadores de la tradición feminista. El abordaje utilizado en este trabajo es desde las perspectivas feministas críticas en Relaciones Internacionales. Primero, problematizamos algunas definiciones de política exterior feministas que circulan, y analizamos críticamente las autodenominadas políticas exteriores feministas de Suecia, Canadá y México, señalando falencias y contradicciones que las atraviesan. En una segunda instancia, procedemos a la elaboración de una definición de PEF que incorpora elementos provenientes de múltiples feminismos (decolonial, autónomo, negro, indígena), y que se asume explícitamente situada desde una perspectiva geográfica y cultural, subalternizada y contrahegemónica. Finalmente, proponemos una gradualidad en la categorización de aquellas políticas exteriores que empiezan a abandonar posicionamientos androcentristas y cisheteropatriarcales, a partir de la construcción de los conceptos de "Politica Exterior con Perspectiva de Género" (PEPG), y "Politica Exterior con Perspectiva de Género y Agenda Feminista" (PEGAF), cuyos significados y diferencias desarrollamos.

\section{Palabras Clave}

Política Exterior Feminista; Política Exterior con Perspectiva de Género; Agenda Feminista; Suecia; México; Canadá; interseccionalidad.

\section{TitLE}

Problematise and Deconstruct the Hegemonic Concept of Feminist Foreign Policy Abya Yala: to a Gradual Purpose in the Implementation

\section{Extended Abstract}

Since 2014 Sweden has had a Feminist Foreign Policy (PEF in Spanish). This concept has been widely used in academic and political circles, without enough progress in its proper definition and delimitation. Scarce theoretical formulations, almost all of them coming from American and/or European academics, fail to provide the improvement of the concept and are limited to a series of minimal characteristics, which are influenced by a liberal and ethnocentric reading of feminism. It allows the

DOI:

https://doi.org//0.15366/relacionesinternacionales202I.48.005

Formato de citación recomendado:

BARBAS, Juan Martín, CHAVES, Dulce Daniela y LUCERO, Mariel Reneé (2022). “Problematizar y deconstruir el concepto hegemónico de Política Exterior Feminista desde Abya Yala: hacia una propuesta de gradualidad en la implementación", Relaciones Internacionales, n 49, pp. 7I-92.
* Juan Martín BARBAS,

Doctorando en Ciencias Sociales (UNGS) y Maestrando en Relaciones Internacionales (UNLP). Licenciado en Ciencia Política (UBA). Miembro de Centro de Estudios en Relaciones Internacionales y Género(s) del IRI (FCJyS, UNLP).

\section{Dulce Daniela}

CHAVES,

Doctoranda

y Magíster en Relaciones Internacionales por la UNLP (Argentina).

Coordinadora del

Centro de Estudios en Relaciones Internacionales y Género(s) del IRI (FCJyS,UNLP).

Mariel Renée LUCERO,

Directora del

Centro de Estudios de Relaciones Internacionales y Medio Ambiente (CERIMA) y profesora en la FCPyS- UNCUyo, y en la UCH (Mendoza-Argentina).

Doctoranda en Relaciones Internacionales por la UNLP

Recibido:

26.05.202

Aceptado:

|6.|1.2021 
notion of PEF to be used to refer to many diverse situations, and to establish, at least symbolically, a sign of equality between cases that have little elements in common; for instance, the foreign policies of Sweden, Canada, and Mexico. This situation is not insignificant, since, on the one hand, it reduces the importance and impact of the proposals that feminism has been developing for decades in the field of international relations theory; and on the other hand, it allows government officials and some intellectuals to appropriate and make superficial use of principles established in the intense struggles that women and other feminized sectors have been involved in for a long time.

Faced with this panorama there is a need to conceptualize. It recovers the most transformative elements of the feminist tradition. To this end, we will problematize some theoretical definitions of PEF, and the self-denominated Feminist Foreign Policies currently in existence, showing the notable weaknesses and contradictions that cross them. We then proceed to the elaboration of a definition of PEF that incorporates elements coming from multiple feminisms (radical, decolonial black, indigenous), and that above all adopts explicitly a geographical, cultural, subalternate and counterhegemonic perspective. Likewise, we propose a gradual categorization of those foreign policies that begin to abandon androcentric and cisheteropatriarchal positioning, from the concepts of Foreign Policy with a Gender Perspective (PEPG in Spanish), and Foreign Policy with a Gender Perspective and Feminist Agenda (PEGAF in Spanish), and whose meanings we shall develop.

Although the concept of PEF could be used for naming a foreign policy that gives a complete and uncontradicted account of the postulates upheld by feminism, we believe that it is more appropriate to use other, less comprehensive concepts. In general terms, the PEF corresponds to a liberal and institutional feminist approach, which underestimates other social actors as legitimate interlocutors. Their strategies are based on a gender mainstreaming approach, perfected in international organizations and replicated by various state entities; as such they ignore the ethnic, cultural, religious and socioeconomic particularities of the populations in which they are applied. In them, divergences between the multiple governmental spheres are not unusual. Moreover, even within the Ministries of Foreign Affairs it is possible to identify notorious incongruities between sectors that are, or are not, crossed by the gender perspective.

Secondly, we suggest the use of the concept of Foreign Policy with a Gender Perspective and Feminist Agenda. Considering that the levels are cumulative, to the previous characterization, we add the importance of substantive representation; the identification and sanctioning of the different forms of violence within and outside national borders; and the need to at least begin to question the heteropatriarchal structures of oppression from a discursive point of view, for which the field of diplomacy is fundamental. This gradually problematizes the meanings and stereotypes disseminated by institutions, understanding that the dispute over women's rights and other sex gender identities must also take place at the symbolic level.

In the Swedish case, we understand that it corresponds to what we have called PEGAF. Indeed, Sweden has done important work concerning development assistance, and the financing of international institutions related to the protection of rights and the empowerment of women. Likewise, of all the cases considered, Sweden is the one in which there is the greatest congruence between its foreign policy and its domestic policy, as well as the work that the Nordic country has been carrying out within the Ministry of Foreign Affairs to consolidate a gender approach. Even from a discursive point of view, the Swedish authorities have questioned certain characteristics of the international system, and have had diplomatic disputes with some countries based on issues related to the violation of human rights. However, none of the above has been sustained over time, and the back and forth has been constant. There have been notable discrepancies in the commitment to a feminist agenda among the different ministries, and in some areas, such as defense, the transformations have been insignificant. In addition, on many occasions, Sweden has abandoned its commitment to the defense of feminist postulates, when obstacles or risks have arisen in relation to the national interest- as defined in androcentric terms. The conservative turn in immigration matters, or the government's refusal to confront the strategic sector of the arms industry, are evidence of this.

Based on the analysis carried out on the case of Canada, we consider that this country can be framed within Foreign Policies with Gender Perspectives (PEPG), since it presents domestic and international antecedents in the work on women's issues. However, the fact that it defines its foreign policy only in one area, such as development assistance, and focuses its empirical work mainly on economic issues, means that it does not achieve the necessary comprehensiveness to place it at the highest level. Likewise, Canada does not make progress in criticizing or questioning, even discursively, the hegemonic global dynamics and institutions, nor does it question its place in the international structure.

Finally, evaluating the Mexican case, we ask ourselves: How can we think of a feminist foreign policy when at the domestic level the drug cartels and human trafficking networks continue to exercise their power and violence with total impunity, and in conjunction with broad sectors of politics, justice and the police? How could the Mexican PEF make sense in a country where people continue to "disappear" or become victims of extrajudicial executions within the framework of democracy, most of the time with representatives of the security forces as the ones responsible? In which part of the gender equality plan can we frame the femicides and transfemicides that place Mexico as one of the most dangerous countries to be a woman or dissident of the heteronorma? Regrettably, we consider that the country is not even in a position to aspire to the lowest level described here -that is,the PEPG- since all its current actions (and those of the last decades) are detrimental to the values and principles that the feminist stance upholds.

The criticisms raised in the three case studies addressed has sought to identify their weaknesses and to construct more appropriate concepts that would point to the different types of external policies that are currently oriented towards women. Moreover, this would make it possible to define them with an appropriate term that is in line with their real actions and not mere rhetoric. Our work, of course, is not limited to a conceptual correction; but, above all, it tries to generate a concrete contribution for the generation of tools and the definition of public policies that have a positive impact on the life of the communities represented.

\section{KEYWORDS}

Feminist Foreign Policy; Foreign Policy with a Gender Perspective; Feminist Agenda; Sweden; Mexico; Canada; interseccionality. 
"La paz no es solamente la ausencia de guerra; mientras haya pobreza, racismo, discriminación y exclusión

dificilmente podremos alcanzar un mundo de paz"

Rigoberta Menchú

I ntroducción

El uso del lenguaje dentro del feminismo no es azaroso. La reafirmación de lo expuesto se traduce en el uso de uno de los métodos más significativos en los estudios feministas, como es el deconstructivismo (Spivak, 1990)'. Este implica una reflexión sobre la utilización de los términos, que va más allá de la identificación del contenido con una definición, poniendo el énfasis en la filiación al marco conceptual y al contexto sobre el cual se asientan dichos conceptos.

Partiendo de esto, consideramos que la banalización en el uso del término "Política Exterior Feminista" (PEF) por parte de gobernantes, académicos/as y comunicadores/as, resulta preocupante. Este abuso del concepto termina vaciándolo de contenido, y es el disparador del presente artículo colaborativo. Por ello, frente a una realidad creciente de casos diversos, se genera la exigencia de elaborar académicamente una definición a partir de características acordes a este nuevo fenómeno, evitando que el término "feminista" pierda su sentido de uso, y se convierta en inocuo y funcional al sistema patriarcal (Goertz y Mazur, 2008).

En este sentido, uno de los primeros problemas que encontramos es la desconsideración frente a la heterogeneidad del movimiento feminista, ya que, se conforma de diversas ramas políticas e identitarias; por cuanto lo asertivo es expresarse en términos de "feminismos", en plural. Cuando los gobiernos hablan de la adopción de una PEF, homogeneizan el feminismo sobre el cual se construye, lo cual evidencia un profundo desconocimiento del tema. Esto es significativo dado que posicionarse desde una u otra corriente feminista, implica un perfil de acercamiento distinto hacia las mujeres, otras identidades sexo genéricas y hacia lo internacional, en las diversas dimensiones política, económica, social y cultural.

Más allá de lo discursivo y de las buenas intenciones que podríamos suponer por parte de algunos/as ideólogos/as de la PEF, queremos explorar en aspectos que consideramos que se contraponen a este concepto. Consideramos que la clave del debate no gira en torno al prestigio mundial que implicaría ser un país regional pionero en posicionarse con una PEF, sino en la coherencia interna y externa que el estado debe demostrar en sus acciones para autodenominarse en esos términos. De otra forma, se corre el riesgo de producir un efecto inverso y provocar el vaciamiento inaceptable del sentido histórico y emancipador de las luchas feministas alrededor del planeta.

Por lo anterior, en este artículo nos proponemos tres objetivos principales: a) reflexionar acerca de lo que consideramos una definición más asertiva del fenómeno, problematizando el concepto hegemónico de PEF; b) indagar comparativamente las experiencias de Suecia, Canadá y México en relación con la PEF; y c) proponer nuevas categorías para analizar la implementación

Si bien Gayatri Chakravorty Spivak se asocia al método deconstructivista feminista, diferentes corrientes y académicas lo utilizan, siendo en las Relaciones Internacionales las más representativas las perspectivas feministas posmodernistas, poscoloniales y decoloniales. 
del fenómeno desde un enfoque gradualista y situado en el Abya Yala ${ }^{2}$.

\section{Deconstruyendo definiciones de Política Exterior Feminista}

Este trabajo aborda la cuestión desde una perspectiva feminista crítica en Relaciones Internacionales, que incluye el feminismo decolonial. A partir de esta óptica, nos referimos a la política exterior como subcampo de las Relaciones Internacionales. En este sentido, ésta se define históricamente en términos androcéntricos y desde una mirada hegemónica; es decir, desde el Norte Global. Como consecuencia de esa génesis, la calificación de "feminista" exige la (re)construcción y el debate de esta novedosa categoría, aprovechando la tensión para generar teoría como herramienta analítica que permita comprender y transformar los ordenamientos, categorías y sistemas de la realidad social (Richard, 20I3).

Desde hace aproximadamente un lustro, al interior de la disciplina de las Relaciones Internacionales se intenta avanzar en la definición del concepto "política exterior feminista". En gran medida, este proceso - y en paralelo, el creciente interés académico en esta cuestión — se originó a partir de la voluntad manifestada por algunos países - el primero de ellos, Suecia a fines del 20l4-, de orientar sus políticas exteriores en función de lineamientos feministas.

En esta línea, uno de los desarrollos teóricos pioneros fue elaborado por Hanna Bäck y Annika Björkdahl (20I7), para quienes una PEF es una política exterior anclada en principios y normas, que busca la inclusión de las mujeres en todos los ámbitos de la actividad humana, con el objetivo de consolidar sus derechos, garantizarles el acceso a recursos, y poner fin a las situaciones de discriminación y violencia a las que se ven expuestas. Christine Alwan y Laurel Weldon (20I7), por su parte, definen a las PEF como un curso de acción orientado hacia actores ubicados fuera de las fronteras nacionales, comprometido con la igualdad de género, el fin de la dominación masculina y la eliminación de los estereotipos.

Estas definiciones primigenias, sin embargo, evidencian varias falencias o limitaciones que dificultan su utilización para el abordaje de los casos de estudio. Por ejemplo, ninguna de las dos profundiza en la relación entre políticas exteriores y políticas domésticas, ni consideran prioritaria la existencia de cierta coherencia entre las mismas. En el caso de la propuesta teórica de Bäck y Björkdahl (2017), se le da una gran relevancia a la configuración que adoptan los discursos sostenidos por las autoridades, perdiendo de vista que no necesariamente se ajustan a las acciones emprendidas realmente.

Además, tanto estas autoras como Alwan y Weldon (2017), parecen quedar atrapadas en una mirada esencialista de las mujeres, lo cual las lleva a definir al sujeto del feminismo en términos biológicos. Esto último, les impide incorporar en sus elaboraciones a otras identidades

\footnotetext{
En el presente artículo elegimos la categoría de Abya Yala, en lugar de la de América Latina y el Caribe, para dar cuenta de las naciones (y cosmovisiones) preexistentes a la ocupación territorial de los colonizadores europeos y al genocidio cometido hacia nativos/as, inferiorizados/ as en razón de su cultura e identidad. Como señala Walter Mignolo (2003), el término América fue la renominalización impuesta a la región a partir del siglo XV en el marco del proceso de colonización. De allí, que siguiendo a Lorena Cabnal (Gargallo, 20I5, p. 26), consideramos que “(e)I paso que necesitamos dar es nombrar desde nuestros propios idiomas liberados y cosmovisiones, las categorías y conceptos que estamos construyendo para el análisis de nuestras realidades históricas de opresión, pero también de liberación, como mujeres indígenas, originarias, campesinas, rurales o de pueblos".
} 
o sectores tradicionalmente feminizados y/o subalternizados. Asimismo, carecen de una crítica de carácter estructural, por lo cual sus propuestas se agotan en la incorporación de mujeres a determinados ámbitos —add women and stir, propio del liberalismo—, y la adhesión a ciertos instrumentos internacionales —en sintonía con un pensamiento meramente institucionalista ${ }^{3}$ -

Algunos de los aspectos mencionados han sido superados por formulaciones más recientes, como por ejemplo, la elaborada por Lyric Thompson y Rachel Clement (2019), quienes sostienen que una PEF es una política estatal que busca definir la interacción con otros estados y movimientos; priorizando la igualdad de género y la consagración de los derechos de las mujeres y otros grupos marginados. La misma debe implicar una asignación significativa de recursos, y buscar una "perturbación" de las estructuras patriarcales y de dominio masculino (Thompson y Clement, 2019). De igual forma, resultan interesantes los planteos sostenidos por Kristina Lunz y Nina Bernarding (2020). Para estas autoras, una PEF debe implicar un cuestionamiento del status quo y un replanteamiento radical de las dinámicas y configuraciones del sistema político internacional.

Tal como puede verse, en ambos casos se incorporan ciertos elementos de crítica estructural, y se amplían los sectores que deberían considerarse representados por la implementación de una PEF, aunque sin que haya una enumeración explícita de estos. No obstante, hay aspectos que continúan sin ser señalados, y que entendemos son de absoluta relevancia. Por ejemplo, no se problematiza la adopción de una PEF por parte de países que ocupan posiciones hegemónicas a nivel global, ni tampoco la desigual distribución de recursos asociados a las jerarquías impuestas por la estructura internacional, que perjudican principalmente a las mujeres y sectores feminizados. Esto probablemente se deba a que las definiciones mencionadas se elaboraron desde una concepción etnocéntrica, territorial y culturalmente situada en los países centrales.

Además, aunque se cuestionan estructuras políticas y militares - la OTAN, por caso- no ven imprescindible profundizar en aspectos económicos propios del sistema capitalista, como son los efectos perniciosos de la economía de libre mercado sobre las poblaciones de los países periféricos, siendo las mujeres y las/os menores de edad quienes más afectadas/os se ven. La crítica a dichos aspectos es inescindible del cuestionamiento a las dinámicas que refuerzan las inequidades globales, como el extractivismo y el libre comercio (Svampa y Slipak, 20I5). Así también con las instituciones que las impulsan y consolidan, por ejemplo, la Organización Mundial del Comercio y el Fondo Monetario Internacional.

A las falencias y críticas sobre estos conceptos construidos desde la academia, podemos mencionar la simpleza de los indicadores que se proponen y la ambigüedad que los convierte en inoperables. En síntesis, por lo analizado y expuesto, consideramos que estas definiciones precedentes resultan insuficientes para enmarcar una PEF. Lo anterior principalmente porque en su amplitud e imprecisión teórica casi cualquier país puede ampararse y autodesignarse como ejecutor de una política exterior feminista.Y, además, porque en esa creencia de "fórmulas exportables" se pierde la importancia de considerar la especificidad respecto a los contextos donde la PEF sería implementada ${ }^{4}$.

Corriente que ha prevalecido en la esfera teórica de las Relaciones Internacionales desde la conformación de la Organización de las Naciones Unidas.

4 Al respecto, consideramos interesante avanzar en posteriores debates en torno a lo que Aggestam y Bergman-Rosamond enuncian como 


\section{Algunos aportes desde donde pensar en clave contrahegemónica}

Como menciona Manuela Mesa (202I), citando a Sanahuja, sólo las teorías feministas en las Relaciones Internacionales plantean las desigualdades sociales en el sistema internacional, contemplando el análisis crítico del carácter epistemológico fundacional del patriarcado. En este sentido, reconocemos que si bien son diversas las corrientes feministas dentro de la disciplina, nos interesa destacar aportes de aquellas que dialogan con nuestra propuesta situada desde Abya Yala.Así, nos posicionamos desde una perspectiva emancipadora, decolonial y descolonizadora 5 .

Para esta perspectiva es fundamental el concepto de interseccionalidad. Originalmente acuñado por Kimberlé Crenshaw en 1989, el mismo ha servido en contextos como el latinoamericano —siguiendo a Mara Viveros Vigoya (2016, p. 7) — para teorizar la "categoría 'mujeres' como clase, producida por un sistema de dominación autónomo e irreductible a las relaciones de producción capitalista”. En definitiva, la autora nos invita a romper con la idea homogénea y universal de una sola forma de concebir a "la mujer", problematizando las diversas trayectorias vitales, a partir de las intersecciones que atraviesan a las distintas identidades en múltiples “experiencias de sexismo" (Viveros Vigoya, 2016, p. 7), clasismo y racismo, que nos ubican como mujeres de un lado u otro en la posición social y de privilegio.

María Lugones (2008), por su parte, sostiene que "la interseccionalidad revela lo que no se ve cuando categorías como género y raza se conceptualizan como separadas unas de otra. La denominación categorial construye lo que nomina" (p. 8I). En línea con lo anterior, Breny Mendoza (20I0, p. 23), repensando a Aníbal Quijano, se refiere a las mujeres del Tercer Mundo y los efectos de la colonización; afirmando que en ese proceso histórico de dominación, estas subjetividades "del mundo colonizado no sólo fueron racializadas sino que al mismo tiempo fueron reinventadas como 'mujeres' de acuerdo a códigos y principios discriminatorios de género occidentales”. Esto implica que la estructura colonial de las sociedades latinoamericanas debe ser considerada parte de las relaciones de poder que se presentan en nuestros territorios; circunstancia que conduce a observar, por ejemplo, la forma de concebir el desarrollo en los pueblos indígenas (ligado a lo comunitario, el "buen vivir" y la naturaleza), contrapuesto al modo occidental, que lo percibe desde una mirada capitalista, individualista y de consumo.

\section{Los casos de estudio: de la propuesta "ideal" al pragmatismo incoherente}

\section{Suecia}

A finales del 2014, tras la llegada al gobierno de una coalición entre socialdemócratas y ecologistas, Suecia se convirtió en el primer país del mundo en sostener explícitamente su compromiso con

“políticas exteriores feministas diferenciadas" (Mesa, 2021, p. 126).

5 Entendemos por decolonialidad el proceso de deconstrucción de los patrones socioculturales, políticos e intelectuales impuestos por la colonización europea sobre las poblaciones nativas de Abya Yala. Dichos patrones, han sido indispensables para el desarrollo de la modernidad capitalista, la hegemonía europea y la imposición de un sistema de jerarquías en virtud de la creación de identidades racializadas. En palabras de Quijano (I992),“(I)a cultura europea u occidental, por el poder político militar y tecnológico de las sociedades portadoras, impuso su imagen paradigmática y sus principales elementos cognoscitivos, como norma orientadora de todo desarrollo cultural, especialmente intelectual y artístico" (p.13). Precisamente, esa colonización del imaginario de los/as dominados/as, es lo que el autor denomina colonialidad. 
una política exterior feminista. A cargo quedó Margot Wallström, comprometida con la defensa de los derechos de las mujeres en diversos ámbitos internacionales. Según las autoridades del país nórdico, la PEF sueca es:

“...un método de trabajo y una perspectiva que parte de tres elementos y se basa en un cuarto. Implica que el Servicio Exterior sueco, en todas sus áreas, debe esforzarse por consolidar los derechos, la representación y los recursos de todas las mujeres y niñas sobre la base de la realidad en la que viven" (Ministerio de Asuntos Exteriores de Suecia, 2019, p. II)

En función de llevar a la práctica esta PEF, se elaboró el Plan de Acción del Servicio Exterior Sueco para una Política Exterior Feminista 2015-2018, luego actualizado con un segundo Plan de Acción para el periodo 2019-2022. Ambos planes se incorporaron al Plan Operacional del Servicio Exterior de Suecia, y establecieron como objetivos prioritarios los siguientes:

a) Impulsar el pleno disfrute de los Derechos Humanos.

b) Combatir toda forma de violencia física, psicológica o sexual sufrida por mujeres y niñas.

c) Aumentar la participación de las mujeres en los procesos de prevención y resolución de conflictos.

d) Garantizar la representación de las mujeres en todos los ámbitos sociales y políticos.

e) Avanzar en el empoderamiento económico de las mujeres.

f) Asegurar el acceso a derechos sexuales y reproductivos.

Además, desde el 2017, se agregó un séptimo objetivo relacionado con la capacitación y formación de los recursos humanos al interior del Servicio Exterior (Ministerio de Asuntos Exteriores de Suecia, 2018).

Al analizar diversos aspectos de la política exterior sueca, es posible hallar múltiples elementos que no se condicen con la aspiración del país nórdico de convertirse en una superpotencia humanitaria. Uno de los más notorios, es el papel que juega en el comercio internacional de armas y equipamiento militar. Según datos del Stockholm International Peace Research Institute (SIPRI) (2020a), los nórdicos se encuentran entre los quince principales exportadores de material bélico, y esta posición no se ha modificado a lo largo del periodo 2014-2020. Con el agravante de que buena parte del mencionado material ha sido adquirido por países con un pobre historial de respeto por los Derechos Humanos, como Arabia Saudí y Emiratos Árabes Unidos (SIPRI, 2019).

Por otro lado, no puede soslayarse el hecho de que Suecia viene experimentando un fuerte proceso de rearme y militarización, según las autoridades, justificado por el aumento de las tensiones con Rusia. En el marco de dicho proceso, el gobierno socialdemócrata ha aumentado notablemente el presupuesto militar (la Ley de Defensa 202I-2025 apunta a un aumento del $40 \%$, el más alto desde la década del cincuenta), lo cual coloca a Suecia en el puesto número 33 de los países con más recursos destinados a las Fuerzas Armadas (SIPRI, 2020b). Asimismo, Suecia fortaleció notablemente sus vínculos con diversas organizaciones de seguridad colectiva, 
siendo el más relevante y polémico, — desde una perspectiva feminista - el que sostiene con la OTAN, la principal alianza militar a nivel global, cuyas intervenciones en terceros países suelen ir acompañadas por frondosas denuncias por violaciones a los Derechos Humanos.

En otro orden, desde finales del 2015 las autoridades suecas han implementado una serie de medidas tendientes a disuadir la llegada de solicitantes de asilo, y dificultar el acceso a la ciudadanía permanente por parte de los inmigrantes extracomunitarios. En un primer momento, en el marco de lo que se conoció como la "crisis de los refugiados", dichas medidas se justifican como necesarias para evitar el colapso de los servicios públicos y el desborde de la capacidad sueca de recepción de extranjeros (Oficina del Primer Ministro de Suecia, 20 I5). Posteriormente, una vez que el flujo inmigratorio se redujo drásticamente, las autoridades mantuvieron las medidas restrictivas y los controles fronterizos, pero modificando el discurso mediante el cual se los buscaba legitimar.

A finales del 2016 el eje estuvo puesto en la necesidad de garantizar la seguridad de la población local, frente a potenciales ataques terroristas (Ministerio de Justicia de Suecia, 2018). Esta postura, que coloca al inmigrante en un lugar de riesgo para la población local, se vio agravada en el marco de la emergencia sanitaria provocada por el virus del covid-19. El principal epidemiólogo del Estado, Anders Tegnell, le indicó a la prensa que el gran número de contagios se debía a la presencia de inmigrantes en el país (Capar, 2020). Y aunque posteriormente afirmó haberse expresado incorrectamente, su exabrupto dejó al desnudo el giro discursivo de la socialdemocracia sueca, cuyo corolario es la imagen del otro como una amenaza.

En consideración de todo lo anteriormente mencionado, numerosas ONG e instituciones internacionales vienen alertando sobre el avance de esa mirada securitaria sobre la inmigración. La cual deja en evidencia la incongruencia entre los postulados de solidaridad y humanitarismo que Estocolmo sostiene desde lo discursivo, y las acciones concretas - de carácter reaccionario y securitario- que ejecutan frente a los inmigrantes y solicitantes de asilo (Amnistía Internacional, 2017; CEAR, 2019).

\section{Canadá}

A diferencia de Suecia, la propuesta canadiense se encuentra orientada a un aspecto particular de la política exterior: la asistencia feminista al desarrollo. Esta perspectiva surgió desde la campaña del Primer Ministro, Justin Trudeau (20I5-...), promoviendo un gobierno paritario. Sin embargo, el contexto de surgimiento se asoció a la postulación de Canadá a ocupar para junio de 2020 uno de los asientos rotativos dentro del Consejo de Seguridad. En este sentido, es oportuno recordar que la promoción de la cooperación al desarrollo es uno de los ítems más relevantes evaluados por Naciones Unidas entre los países candidatos.

Esta política se implementó desde 2017, teniendo al frente a la Ministra de Desarrollo Internacional y de la Francofonía, Marie-Claude Bibeau (2015-2019) y a la Ministra de Asuntos Exteriores, Chrystia Freeland (2017-2019). El documento declara un aspecto específico orientado a las mujeres como es la Política Feminista de Asistencia Internacional (FIAP, por sus siglas en inglés). Allí promueven: 
a) La igualdad de género y el empoderamiento de niñas y mujeres.

b) La dignidad humana.

c) El crecimiento de todos.

d) El medio ambiente y la acción climática.

e) La gobernanza inclusiva.

f) La paz y seguridad (Global Affairs Canada, 20I7b).

La política exterior canadiense orientada a la asistencia al desarrollo de las mujeres, deja en su accionar al descubierto significativas contradicciones, y obliga a interpelar sobre los objetivos reales propuestos por el "modelo" en estos cuatro años. Lo primero que es posible observar es la falta de precisión respecto al tipo de feminismo adoptado, dentro del documento de declaración de la FIAP.

En este sentido, coincidimos con Laura Parisi (2020), e identificamos que este asistencialismo al desarrollo enmarcado bajo el paraguas de Naciones Unidas - como claramente queda destacado en cada ítem del documento informativo de la FIAP - convive entre un feminismo neoliberal y un neoliberalismo feminista ${ }^{6}$. Al respecto, en el ítem específico referido a la seguridad sostiene que "la Agenda 2030 reconoce que no puede haber un desarrollo sostenible sin paz, y no hay paz sin desarrollo sostenible", dejando en evidencia la línea de pensamiento que concibe a la seguridad ligada a lo económico, e invisibiliza a las violencias como un factor de obstaculización (Global Affairs Canada, 20I7b, p. 56).

En otras palabras, la interrelación entre paz, seguridad y el desarrollo sostenible mantiene, según la FIAP, una concepción de causalidad directa que entiende que a la paz se llega por vía del desarrollo económico, entendido en términos de aumento de la circulación monetaria y participación en el mercado, en consonancia con el Objetivo de Desarrollo Sostenible 16 sobre paz, justicia y fortalecimiento de las instituciones. Por otro lado, sostiene que con la inclusión de mujeres en los procesos de construcción de la pacificación, además hay mayor probabilidad de que estos sean exitosos (Global Affairs Canada, 20l7b). Esta interpretación canadiense de causalidad directa desconoce un aspecto central para el feminismo que entiende que la ausencia de violencia bélica no libera a las mujeres, $y$ a la sociedad de otras violencias, que son continuas y muchas veces más graves; incluso muchas de ellas se dan en el ámbito privado, como son las sexuales y de explotación laboral, perpetradas por el sistema patriarcal.

Desde una metodología feminista, la interpretación y el uso del lenguaje resultan centrales en el análisis, por eso la enunciación de la paz y la seguridad al final del listado no resulta casual. La ambigüedad en el texto de la FIAP, cuando refiere a empoderar a las mujeres es en el desarrollo económico, y soslayar cuestiones centrales, como son los derechos laborales (condiciones de trabajo, lactancia, acoso laboral, etcétera) y las brechas de género en los salarios, entre otras cuestiones.

Por otra parte, los indicadores claves de rendimiento en el ítem sobre seguridad y paz

\footnotetext{
6 Parisi define el neoliberalismo feminista como promotor de la igualdad de género "necesario y bueno para el crecimiento económico, y el feminismo neoliberal promueve la ciudadanía de mercado como el camino primario para adquirir la igualdad de género" (2020, p. 2, traducción propia).
} 
escogidos por Canadá, sólo consideran la cantidad de peacekeepers (enviados/as a las misiones de paz), y las inversiones en programas relacionados al desarrollo económico antes que la medición del impacto en los resultados sobre las mujeres del territorio al que se envió la misión. Esto destaca la indiferencia en analizar la diversidad regional, contextual y cultural, es decir, incluir la interseccionalidad, orientado hacia mujeres en su heterogeneidad.

A nivel global, entre las críticas más fuertes que resuenan respecto de la Resolución I325, es que después de $2 \mathrm{I}$ años de existencia, se mantiene un bajo porcentaje de participación de mujeres en la mediación en conflictos bélicos. En este sentido, Canadá es uno de los países promotores del concepto de seguridad humana y ayuda humanitaria. Como sostienen Heather Smith y Tari Ajadi (2020), la promoción de ambos conceptos —el de seguridad humana y el de FIAP — presentan similitudes. Se encuentran construidos sobre valores liberales, restringidos a la retórica propositiva y de marketing político, de convertirse en el paladín de la igualdad de género a nivel internacional, subido a la cresta de la moda de "la agenda discursiva mediática" antes que de accionar profundas transformaciones sobre el sistema patriarcal (Smith y Ajadi, 2020, p. 368).

Por otro lado, entre los cinco primeros países que desde 2016 reciben ayuda asistencial de Canadá, dos de ellos - Bangladesh yAfganistán - también mantienen un comercio en equipamiento militar que se compone, entre otras cosas, de armamento pequeño y ligero (Global Affairs Canada, 20 I6; 2017a; 2018;2019). Según Naciones Unidas (2018), las armas pequeñas y ligeras están identificadas como peligrosas para la seguridad de las mujeres, niños y niñas, sobre todo en el ámbito privado y relacionado a la violencia sexual y doméstica. En ese sentido, cerca de un tercio de los feminicidios se cometen con este tipo de armas, $y$ favorecen el incremento del reclutamiento de menores en los casos de conflictos armados.

Además, Canadá ocupa el puesto 16 en exportación de armas a nivel mundial desde 2015 , detrás de Suecia. Entre algunos de sus principales clientes, se encuentra un país con serios antecedentes contrarios a los Derechos Humanos por su accionar en la zona de Gaza, como es Israel, y otro que agrega la discriminación a los derechos de las mujeres en particular, como es Arabia Saudí (Global Affairs Canada, 2019). Se construye así una dinámica perversa de ventas de armas, acompañada por asistencia al desarrollo o a la reconstrucción.

Finalmente, una de las críticas más contundentes sobre la efectividad de la FIAP es el presupuesto destinado a asistencia que se mantiene por debajo del promedio histórico canadiense. Hacia 2020, mantenía su cifra de cooperación internacional por debajo del desempeño histórico del país, según el propio Canadian Council for International Cooperation $(\mathrm{CClC} / \mathrm{CCCl})$, ahora Cooperation Canada. En este sentido, Canadá desde la llegada de Trudeau al poder, destina un porcentaje a la asistencia del $0,26 \%$ del PBI, por debajo del promedio de ayuda histórica del $0,7 \%$ del PBI, y de lo sugerido por Naciones Unidas. En otras palabras, por un lado, fomenta el empoderamiento de las mujeres y niñas, pero mantiene bajos los recursos destinados para alcanzar tales propósitos. Esto pone de manifiesto la contradicción existente entre la promoción de un feminismo fuerte pero imposible de alcanzar sin recursos, con un "presupuesto miserable” (Morton et al, 2020, p. 33I). 


\section{Estados Unidos Mexicanos}

En el caso de México, cabe señalar que en enero del 2020 se anunció la adopción de una política exterior feminista, que se implementará durante el período 2020-2024; convirtiéndose de este modo en el primer país de América Latina y el Caribe en posicionarse desde dicha política. Tal como señala Martha Delgado Peralta (2020, pp. I34- I35), Subsecretaria para Asuntos Multilaterales y Derechos Humanos del país del norte, la misma se basa en cinco ejes:

a) Una política exterior con perspectiva de género y una agenda feminista en la que México tome un papel internacional de liderazgo en los temas de la agenda multilateral, así como en la política exterior regional y bilateral, que tenga impacto en la igualdad sustantiva de género.

b) Paridad en cargos y una reforma organizacional para lograr la equidad en el ámbito profesional.

c) Una Secretaría de Relaciones Exteriores que enfatice la acción colectiva para crear un espacio laboral sin acoso ni violencia por motivos de género.

d) Hacer visibles los liderazgos femeninos y llamar la atención sobre las contribuciones de las mujeres a la política exterior.

e) Un enfoque interseccional en todas las posiciones, estrategias y acciones de política exterior.

En el caso mexicano, es imprescindible atender los aspectos domésticos de la seguridad; ya que, como veremos, es un área que condiciona y altera las trayectorias vitales de la ciudadanía, principalmente de los grupos vulnerabilizados.Además, en un contexto de globalización como el que estamos asistiendo, los fenómenos locales terminan traspasando fronteras, transnacionalizándose.

De acuerdo a la información sistematizada por US News \& World Report, en el 2020 Best Countries Rankings, para la categoría "Calidad de vida”, la percepción de seguridad en México es de 0, I\% (US News \& World Report, 2019). Otro dato significativo es el que arroja el "Índice Mujer, Paz y Seguridad" de 2019/20, elaborado por Georgetown Institute for Women, Peace and Security y por The Peace Research Institute Oslo (2019), que ubica a México en el puesto I03 a la hora de analizar las ventajas y desventajas de ser mujer en dicho país.

Otros aspectos del contexto mexicano que están lejos de poder interpretarse desde una perspectiva de género de la seguridad son: el podio que ocupa el país americano junto a Tailandia y Camboya en materia de turismo sexual infantil7; la impunidad legal para los culpables de feminicidios y transfemicidios; la connivencia entre los distintos poderes del estado para garantizar la corrupción de los grupos mafiosos; las constantes amenazas de muerte ${ }^{8}$ y crímenes concretados a periodistas que investigan estas redes delictivas ${ }^{9} ; \mathrm{l}$ la criminalización de la pobreza y de la etnicidad.

En la esfera de política exterior, desde el año 2020 y hasta el 2022 México es uno de los

De la mano de la connivencia entre las Fuerzas de Seguridad, el sistema judicial y político.

8 El ejemplo más conocido es el de la periodista de investigación, Lydia Cacho. Está amenazada de muerte y vive desde hace años fuera de su país natal porque las autoridades mexicanas no pueden garantizar su seguridad. Como bien sostiene ella, el mensaje es que el Estado Mexicano no puede proteger a nadie que desafíe a los sectores mafiosos.

9 Según el Committee to Protect Journalists (2020), que investiga los ataques contra la prensa a nivel global, nueve periodistas fueron asesinados (ocho varones y una mujer) en México en 2020 ( 5 con motivos confirmados y 4 sin confirmar), lo que eleva el número de muertes a II6, desde el año 2000 hasta el 2020. Esto ubica al país del norte en la zona más peligrosa para ejercer el periodismo, excluyendo únicamente las zonas de guerra. 
diez miembros no permanentes del Consejo de Seguridad de Naciones Unidas. Considerando que este órgano, uno de los principales de la ONU, tiene entre sus funciones "mantener la paz y la seguridad internacionales de conformidad con los propósitos y principios" (Consejo de Seguridad, 202I) de la organización, es particularmente alarmante y cínico advertir que dicho país no acate lo prescripto en el Protocolo para prevenir, reprimir y sancionar la trata de personas, especialmente mujeres y niños, que complementa la Convención de las Naciones Unidas contra la Delincuencia Organizada Transnacional, del 15 de noviembre de $2000^{10}$. Consideramos que en tanto a nivel doméstico los Estados Unidos Mexicanos no puedan acabar con este movimiento esclavizador que oprime a mujeres y niña/os en su territorio, es de una doble moral enunciar que lideran un proyecto emancipador feminista hacia el mundo.

En otro orden, un detallado informe publicado en diciembre del 2020, fruto del trabajo conjunto de Data Cívica, EQUIS Justicia para las Mujeres e Intersecta, deja en evidencia que las Fuerzas Armadas mexicanas se encuentran incumpliendo con las capacitaciones en Derechos Humanos y género que la Corte Interamericana ordenó para evitar la repetición de aberrantes crímenes $^{\prime \prime}$; sino que, además, se manifiesta una preocupación por la falta de reglas claras y límites para el proceder del personal que integra dichos espacios de poder. Un ejemplo de ello es que, tal como denuncia "Falsas salvaguardas" (2020), la Secretaría de la Defensa Nacional, "ha gastado cientos de millones de pesos en actividades de capacitación de las que responde no tener registro alguno" (p. 6).

Por otro lado, debemos señalar los negociados internacionales en el ámbito de la industria alimentaria. Según revela una investigación realizada por Kennia Velázquez (202I), y presentada en el portal Sinembargo.mx, empresas transnacionales como Coca-Cola, Pepsi, Danone, Nestlé, Bimbo, entre otras,"extraen anualmente 133 mil millones de litros de agua para producir comida y bebida que no sólo afectan la salud de los consumidores, sino que además provocan serios daños ambientales en México", en un país donde el $24 \%$ de los hogares no tienen agua diariamente.Ante un problema grave alimenticio de la población, un impacto ambiental alarmante, un preocupante estrés hídrico y una ineficiente capacidad - por parte de la Comisión Nacional del Agua (CONAGUA) - para controlar que las grandes empresas cumplan con el volumen autorizado de extracción, México pondera los negocios de capitales extranjeros ${ }^{12}$ sobre el bienestar de su ciudadanía.

Es urgente que el derecho humano al agua potable y a un sistema alimentario sostenible y accesible para todos los sectores socioeconómicos esté por encima de negocios millonarios que afectan el buen vivir de las comunidades. Esto, sin dudas, debe ser considerado un interés nacional, que debería ir en congruencia con los valores de la PEF.

10 En este sentido, la negativa fama mediática que tiene el estado de Tlaxcala tiene como actividad principal la trata de personas y constituirse como "la cuna de los padrotes". Estos traficantes — principalmente- de mujeres con fines de explotación sexual, lideran redes delictivas con conexión al resto de las regiones mexicanas, así como también a Estados Unidos y Europa. Este nivel de proxenetismo sería imposible sin la complicidad de la policía federal, estatal y municipal, los militares, así como de sectores de la justicia y política local y nacional.

"Nos referimos a los siguientes casos: Fernández Ortega y otros vs. México (Fecha de última resolución: 25 de noviembre de 20 I0); Rosendo Cantú y otra vs. México (Sentencia: 3 I de agosto de 20l0); y Alvarado Espinoza y otros vs. México (Sentencia: 28 de noviembre de 2018 ). Esta última sentencia figura en la página oficial de la Secretaría de Defensa Nacional, en la categoría “Acciones y programas” (2020).

12 Y ni siquiera lo hace de modo razonable; pues, de acuerdo con el "informe sobre violaciones a los derechos humanos al agua potable y al saneamiento en México publicado en 2018, se señala que Coca Cola FEMSA pagó 2 mil 600 pesos al año por cada una de sus concesiones" (Velázquez, 202I). Un monto totalmente desproporcionado para las infinitas ganancias que genera la empresa. 
Por último, nos parece fundamental dar cuenta de la importación de armas que el país lleva a cabo; aspecto ligado con lo mencionado previamente en seguridad, sobre la militarización del territorio y la acumulación de poder en manos de las Fuerzas Armadas. Según un informe realizado por ocho organizaciones internacionales ${ }^{13}$, publicado en diciembre de 2020 , y basado en una revisión de documentos militares mexicanos secretos, "las empresas de armas con sede en Europa e Israel exportaron más de $\mathbf{2 3 8 . 0 0 0 ~ a r m a s ~ d e ~ f u e g o ~ a ~ M e ́ x i c o ~ p a r a ~ u s o ~ d e ~ l a ~ p o l i c i ́ a ~}$

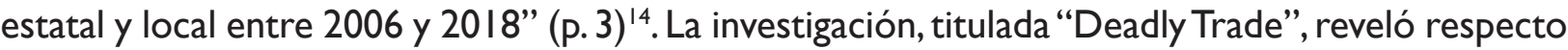
del ejército mexicano que "ninguna otra nación del mundo concentra tanta autoridad para la adquisición, distribución y control de armas pequeñas en una sola institución militar" (p. 6) $)^{15}$.

En síntesis, con diferencias en la forma de implementación en los casos abordados, podemos extraer algunas de las siguientes críticas comunes: en primer lugar, la ausencia de cuestionamientos a las estructuras económicas, jurídicas, políticas y sociales que mantienen la situación de marginación y subyugación de las mujeres y otras identidades subalternizadas, tanto a nivel doméstico como internacional. En segundo lugar, las PEF analizadas mantienen una perspectiva etnocentrista, con una mirada esencialista de "la mujer" en la selección de las problemáticas a priorizar y en el abordaje de posibles soluciones a esas cuestiones, eludiendo las interseccionalidades.

En línea con lo anterior, la ausencia de otros y otras en las políticas propuestas presentan una mirada excluyente de las alteridades sexo genéricas oprimidas que difieren del modelo cisheteropatriarcal $^{16}$. En cuarto lugar, advertimos una omnipresencia del institucionalismo, que identifica como únicos interlocutores válidos en las relaciones internacionales a los estados y organismos internacionales y/u ONG, dejando de lado otros actores y actrices del sistema internacional. Finalmente, la concepción subyacente en las PEF estudiadas se encuentran en concordancia con los valores neoliberales cuestionados por sectores mayoritarios del movimiento feminista.

\section{Hacia una definición propia y situada de Política Exterior Feminista}

Utilizar la palabra feminista como elemento adjetivador del sujeto "política exterior" implica, mínimamente, encontrar presentes algunas características y valores relacionados a ese término. En este sentido, entendemos la importancia de deconstruir el concepto "política exterior feminista", definido hasta el momento desde una posición hegemónica, universalizante; y avanzar en la elaboración de una definición propia, inspirada en el abordaje de los estudios de caso presentados y los postulados fundamentales del feminismo crítico en las Relaciones Internacionales, en especial

13 Global Exchange (Estados Unidos), Vredesactie (Bélgica), OPAL (Italia), Agir pour la Paix (Bélgica), American Friends Service Committee (Israel), Ohne Rüstung Leben (Alemania), NESEHNUTí (República Checa), Comisión Mexicana de Defensa y Promoción de los Derechos Humanos, y el Centro de Estudios Ecuménicos (México).

14 Traducción propia.

15 Traducción propia.

16 En esta categoría compuesta, con el prefijo “cis”, queremos aludir de forma crítica al posicionamiento hegemónico de normalización de la identidad "cisgénero" - que alude a aquellos casos en los cuales la identidad de género tiene concordancia con el sexo que le asignaron a la persona al nacer- en detrimento de identidades trans. Al respecto, y tal como afirman Lelya Troncoso y Valentina Stutzin (20I9, pp. I819), “(I)os puntos nodales de la defensa del orden heteropatriarcal se basan en: (I) la superioridad moral del matrimonio heterosexual, (2) patologización de las personas no heterosexuales, (3) defensa de la familia heterosexual reproductiva como la única forma legítima de familia, y el deber del estado de defenderla en pos del bien social; y (4) la ley natural como orden moral universal que se ve amenazado". 
de sus vertientes situadas en el sur global.

En primer término, consideramos poco confiable a nivel internacional, e irresponsable a nivel doméstico, reclamar una PEF cuando internamente no se alcanzan estándares mínimos de defensa de los derechos de las mujeres y las niñas, ni se promueven acciones favorables para lograr la igualdad de género y la erradicación de violencias contra todas las diversas identidades sexo genéricas. Entendemos que resulta incongruente e ineficaz cuando es sostenida por un país que en el plano interno no logra garantizar la protección básica de los Derechos Humanos.

Dicho en otras palabras, consideramos que debe existir una coherencia entre el comportamiento del estado en el plano internacional y su desempeño en el ámbito nacional. En consecuencia, entendemos que una PEF debe darse en un contexto de transversalización de las cuestiones de género en la totalidad de las dependencias gubernamentales; ya que sería inconsistente que solamente se defiendan postulados feministas desde un único ministerio u organismo estatal.

Una PEF requiere, como condición necesaria pero no suficiente, que se garantice dentro de todos los ámbitos de planificación, ejecución y evaluación de políticas, la representación de las mujeres y otros grupos marginalizados por cuestiones étnicas, religiosas, de clase o de orientación sexual. Esto implica, en primer lugar, asegurar la igualdad de género al interior de los Ministerios de Relaciones Exteriores, así como también la fehaciente capacitación y concientización de todo el personal. Por ello, una PEF se debe acompañar con acciones concretas que favorezcan el acceso y la permanencia de las mujeres y otros sectores excluidos del ámbito diplomático, como personas del colectivo LGBTIQ+.

Además, consideramos imperante en el marco de una PEF la necesidad de nombrar y reconocer a diversos grupos tradicionalmente vulnerados, personas que integran el colectivo LGBTIQ+, personas con discapacidad, identidades afrodescendientes, campesinas, originarias, migrantes, minorías étnicas y/o religiosas. Como bien sabemos las feministas, la enunciación se inscribe como urgencia en un contexto de disputa de sentidos políticos, que luego devendrá en una toma de decisión respecto a dónde invertir recursos, políticas públicas y programas. Las generalizaciones y los conceptos ligados a la universalidad y los metarrelatos, terminan por invisibilizar experiencias y subjetividades diversas que deben ser reconocidas y atendidas en sus demandas específicas.

En otro orden, consideramos que una PEF debe desaprobar la explotación económica y toda forma de extractivismo, en especial de bienes comunes, que implican la explotación del ambiente $y$, por ende, que tienen un impacto negativo sobre las comunidades. La connivencia entre estados y empresas transnacionales que destruyen territorios por proyectos multimillonarios de megaminería y de hidrocarburos, forma parte de un obstáculo para actuar desde la perspectiva feminista. La contaminación del aire y del agua son sólo algunas de las consecuencias de estos negocios que atentan contra la naturaleza y la vida de los/las habitantes de las zonas afectadas. En este sentido, una PEF debe defender la seguridad y la soberanía alimentaria de los países, cuestionando las vigentes prácticas del llamado agronegocio, sobre las cuales se expande la industria alimentaria transnacional. 
Asimismo, entendemos que una PEF debe tener una postura implacable en torno a los delitos que conforman el crimen organizado internacional, ya que constituyen una fuente de dolor y opresión hacia niñas/os, mujeres y otras identidades feminizadas. Como es sabido, la trata de personas con fines de explotación sexual y laboral, las redes trasnacionales de pederastas y la pornografía generada como resultado de coerción hacia sus protagonistas, constituyen grandes derrotas para una comunidad de naciones que dicen proteger a sus representados/as. No incluir la dimensión de la sexualidad en una PEF, significa congraciarse con quienes siguen haciendo de nuestros cuerpos y desigualdades un negocio de carácter global. Sin dudas, una de las caras modernas de la esclavitud es la explotación sexual, a la que algunos sectores pretenden disfrazar de libre albedrío.

Un PEF debería basarse en una perspectiva interseccional, y además, en un enfoque multicultural; ya que consideramos que sólo una política que respete e integre la diversidad sociocultural que configura nuestros países, que reconozca su historia y su valor, puede definirse como feminista. El feminismo desde el que nos posicionamos, además de anticolonial es antirracista; por lo cual, busca romper con el relato único y que se escuchen las voces de quienes fueron convertidas desde la colonización, subalternizadas por un imaginario racista y etnocéntrico.

Tal como sostuvieron las mujeres indígenas y afrodescendientes en la Undécima Conferencia Regional sobre la Mujer de América Latina y el Caribe, organizada por la CEPAL en el 2010, es fundamental reconocer que son "sujetas de derechos", según la Declaración de las mujeres indígenas y afrodescendientes de América Latina, el Caribe y la diáspora (CEPAL, 20l0). Aquí el feminismo decolonial reorienta las miradas sobre aquellas mujeres que doblemente se encuentran marginadas y reconoce lo que sostiene Lugones (2008) acerca de que aunque "en la modernidad eurocentrada capitalista, todos/as somos racializados/as y asignados/as a un género, no todos/as somos dominados/as o victimizados/as por ese proceso. El proceso [colonial] es binario, dicotómico y jerárquico" (p. 82).

Hablar de opresión patriarcal y subordinación colonial implica referenciar la interseccionalidad racial, étnica, de clase, de género y sexualidad; y conduce a mencionar la necesidad de una política anticapitalista. Las estructuras que sostienen la desigualdad global se basan en un conjunto de creencias sobre supuestas jerarquías biológicas, raciales, nacionales, culturales, cisheteropatriarcales; y además, sobre una división sexual del trabajo que reproduce un statu quo esencialista, oprime y genera una distribución inequitativa de la riqueza en detrimento de las mujeres, las disidencias sexo genéricas y el resto de las subjetividades vulnerabilizadas por el estrato social al que pertenecen.

Consideramos que una política exterior feminista es aquella que teoriza y lleva a la práctica acciones emancipatorias para actores y actrices vulnerabilizados/as del escenario global, sin desconocer el contexto macroeconómico y cultural en el cual se inscriben las desigualdades que se intentan eliminar. La PEF debe apuntar a transgredir toda moral religiosa, política y/o social que violente a las mujeres, adolescentes, niñas/os y al resto de las identidades no hegemónicas.

Uno de los tópicos más relevantes en la política exterior de cualquier país es el de la 
seguridad y la defensa. De allí, que resulte fundamental para quienes desean comprometerse con el sostenimiento de una PEF, el lograr delinear políticas de seguridad y defensa acordes con una perspectiva que prioriza la seguridad humana sobre la seguridad estatal,y en la cual la militarización y el ejercicio de la violencia no se consideran instrumentos legítimos para la resolución de los conflictos. Esto implica, en primer término, deconstruir el modo androcentrista y heteropatriarcal desde el cual se define la idea misma de amenaza.

Por último, una PEF debe romper con la dinámica estatocéntrica de las relaciones internacionales que aún mantiene el paradigma tradicional preponderante, e incorporar como interlocutores/as válidos/as y necesarios/as a actores y actrices de la sociedad civil -ONG, movimientos sociales, etcétera-, tanto en el propio país como en el extranjero. A su vez, debe contemplar la existencia de mecanismos que permitan la participación ciudadana y el control democrático sobre las instancias de planificación, ejecución y evaluación. No actuar de esta manera, implica perder de vista que los estados son los representantes de los intereses de los sectores dominantes, que históricamente han excluido a las mujeres y otras feminidades.

En función de las características previamente señaladas y descriptas, entonces, definimos a una Política Exterior Feminista como el conjunto de decisiones y acciones que toma un gobierno, en coherencia con una política externa e interna, que se rige por una perspectiva feminista, de diversidad e interseccionalidad; ponderando las estrategias y los discursos que contribuyen a la emancipación económica, social, política y cultural de las mujeres y subjetividades que integran el colectivo LGBTIQ+, así como también las personas con discapacidad, las identidades afrodescendientes, campesinas, originarias, migrantes, minorías étnicas y religiosas; y que promueven relaciones bi y multilaterales recíprocas, sin jerarquías y de carácter horizontal entre los grupos sociales, y entre los estados.

El país que ostente una PEF requiere de un autocuestionamiento constante del espacio de poder que su estado representa en el sistema internacional; actuar contra las expresiones de violencias que tienen como finalidad la subyugación, marginación y/o exclusión de otros y otras; ponderar la representación paritaria como condición necesaria, pero no suficiente para alcanzar la igualdad; combatir los delitos internacionales, generalmente asociados al crimen organizado transnacional, donde la trata de personas es una de las mayores atrocidades y pone en escena el cuerpo como "territorio político" (Gómez Grijalba, 2013); y guardar una congruencia entre sus principios y los que se rigen en la política doméstica, con su expresión en los Ministerios y Secretarías de las diversas áreas que el interés nacional ha definido.

La cualidad principal de la PEF debería ser el enfrentamiento contra las estructuras que sostienen las “múltiples opresiones" (Lugones, 202I) vigentes en el sistema internacional hacia el cual dirige sus acciones y esfuerzos. En consecuencia, la condición sine qua non es que sea inclusiva, anticapitalista, antimilitarista, anticolonial, antirracista, antixenófoba y "antinacionalista" (Curiel Pichardo, 202I). Respecto a este último punto, entendemos que debe asentarse sobre una base internacionalista, independiente de las limitaciones impuestas de forma tradicional por la soberanía territorial de los estados nación, considerando que intenta impactar principalmente sobre las vidas de las “mayorías feminizadas” (Gargallo, 2009, p. 92). 


\section{Apuntes para una propuesta de gradualidad}

Robert Dahl, uno de los principales referentes teóricos de la Ciencia Política, centró su trabajo en el estudio y la caracterización de los sistemas democráticos. Según este académico norteamericano, ningún régimen político existente -en el presente o en el pasado- puede ser considerado estrictamente una democracia —en tanto gobierno para el pueblo por el pueblo-, ya que para esto deberían cumplirse una serie de condiciones de un altísimo nivel de exigencia (Dahl, 2004).

Por esta razón, y para evitar errores analíticos o vaciamientos conceptuales, propone diferenciar entre las democracias ideales — plenas, pero solamente existentes en la teoría-, y las democracias reales -necesariamente portadoras de algún grado de imperfección-. A estas últimas, sugiere llamarlas "poliarquías". El concepto de poliarquía apunta a lo realmente existente, y constituye una definición empírica y procedimental de estos regímenes políticos (Dahl, 2004). Este concepto, sirve para distinguir las democracias reales de las pseudodemocracias y los regímenes autárquicos, pero sin caer en apreciaciones idealizadas y carentes de sustento fáctico. Las poliarquías se pueden acercar en mayor o menor medida al ideal democrático, en función del grado de cumplimiento que tengan de las diversas características que definen al mismo.

Extrapolando estas reflexiones y propuestas al tópico que nos convoca, consideramos que el concepto de PEF debería reservarse para nombrar una política exterior que dé cuenta de forma acabada y sin contradicciones de los postulados sostenidos por el feminismo, algo que difícilmente pueda darse en las condiciones actuales, y mucho menos en países que ocupan un lugar privilegiado dentro de la estructura internacional, como es el caso de Suecia y Canadá. Para evitar caer en un uso excesivo e inexacto de dicho concepto, y poder nombrar las políticas exteriores actualmente existentes que se autodefinen como feministas o tienen pretensión de serlo, creemos que es más adecuado la utilización de otros conceptos, menos abarcativos.

En tanto una PEF presupone el cumplimiento de una serie de requisitos difícilmente alcanzables en el marco de las actuales configuraciones políticas y económicas internacionales, proponemos la utilización de otros conceptos que permitan nombrar e identificar los casos actuales o potenciales. Dichos conceptos, nos permiten establecer además una gradualidad en la implementación, basada en el cumplimiento de un mayor o menor número de las características atribuidas previamente a las PEF.

En primer lugar, proponemos denominar como Políticas Exteriores con Perspectiva de Género (PEPG), a aquellas políticas exteriores en las cuales se reconoce explícitamente la existencia de desigualdades de género, y se impulsan acciones que buscan una mayor presencia y representación formal de las mujeres en las instituciones internacionales, sin que haya un cuestionamiento - siquiera discursivo- de las estructuras sobre las cuales se asientan el heteropatriarcado, las desigualdades sociales y los distintos tipos de violencias.

En líneas generales, las PEPG se corresponden con un enfoque feminista liberal e institucional, que subestima a otros/as actores/actrices sociales como interlocutores/as legítimos/ as. Sus estrategias se basan en un enfoque de gender mainstreaming, perfeccionado en organismos internacionales y replicado por entidades estatales diversas, desconociendo las particularidades 
étnicas, culturales, religiosas y socioeconómicas de las poblaciones en las cuales se aplican. En ellas, no son extrañas las divergencias entre las múltiples esferas gubernamentales; e incluso al interior de los Ministerios de Relaciones Exteriores, es posible identificar incongruencias entre sectores que están atravesados o no por la perspectiva de género.

En segundo lugar, sugerimos la utilización del concepto Política Exterior con Perspectiva de Género y Agenda Feminista (PEGAF). Considerando que los niveles son acumulativos, a la caracterización anterior, le sumamos la importancia de la representatividad sustantiva; de la identificación y sanción de las distintas violencias al interior y exterior de sus fronteras nacionales; y la necesidad de, al menos, comenzar a cuestionar las estructuras heteropatriarcales de opresión desde lo discursivo, para lo cual el ámbito de la diplomacia es fundamental.Asimismo, esta instancia problematiza de forma gradual los sentidos y estereotipos que se difunden desde las instituciones, comprendiendo que la disputa por los derechos de las mujeres y otras identidades sexo genéricas debe darse también en el plano de lo simbólico.

Lo anterior se enmarca en la necesidad de incluir, como parte de los/as agentes sociales que construyen estas políticas exteriores, a las identidades y comunidades que a lo largo del tiempo estuvieron excluidas de los proyectos de los estados nación latinoamericanos; en tanto podríamos inferir que no representan/ban al/la ciudadano/a modelo ideado por la modernidad. Esta consideración es imprescindible en la PEGAF, no sólo como parte de una reivindicación histórica de aquellas voces, luchas y cosmovisiones que la colonización silenció y violentó; sino también como forma de conciliar los valores e intereses de todos los/as actores/as implicados/as, a fin de lograr una coexistencia armoniosa.

A pesar de ciertas virtudes, en las PEGAF la emancipación económica se mantiene desde una lógica capitalista de mercado, la visibilización es en términos de cuantitatividad, y está limitado el diálogo respecto a actores/actrices no estatales u otros organismos no hegemónicos. Asimismo, no objetan las principales instituciones políticas, militares y económicas internacionales (OMC, Banco Mundial, Consejo de Seguridad, etc.), ni avanzan con acciones concretas en su cuestionamiento sobre el carácter estructural de la opresión y la marginación de determinados grupos sociales. Impera en las mismas, a su vez, una lógica instrumental, según la cual adoptar un enfoque feminista es conveniente, por ejemplo, porque permite la expansión de los mercados o contribuye a una mayor estabilidad en los procesos de paz.

En ambas instancias, tanto las PEPG como las PEGAF, pueden ser sectoriales o transversales. En el primer caso, el estado solamente se apega a lineamientos de género o feministas en unas pocas áreas de su política exterior; mientras que en la mayoría de las esferas se comporta de forma tradicional o androcentrista. En el segundo caso, las políticas de género o el impulso de una agenda feminista influyen en amplias áreas de la política exterior, aunque permanecen al margen de los mismos en los ámbitos tradicionalmente masculinizados, siendo el caso más paradigmático el de la seguridad y la defensa. 


\section{Reflexiones finales}

Tal como señalamos al comienzo del presente artículo, el constante uso del concepto PEF está dando lugar a un vaciamiento de su contenido. Por otro lado, resulta poco lógico que una misma categoría teórica se utilice para etiquetar realidades concretas absolutamente dispares, como por ejemplo la de Suecia y México. De allí, la importancia de incorporar otros conceptos — de menor alcance-, que permitan una gradualidad en las definiciones, más ajustadas a la evidencia empírica. En base a dicha propuesta conceptual, procederemos entonces a clasificar los casos de estudio seleccionados.

En el caso sueco, entendemos que se corresponde con lo que hemos denominado como PEGAF. En efecto, Suecia ha desplegado una importante labor en relación con la asistencia al desarrollo, y el financiamiento de instituciones internacionales relacionadas con la protección de derechos y el empoderamiento de las mujeres. Asimismo, de todos los casos considerados, el de Suecia es aquel en el cual existe una mayor congruencia entre su política exterior y su política doméstica, así como también debe mencionarse el trabajo que el país nórdico viene realizando al interior del Ministerio de Asuntos Exteriores para consolidar un enfoque de género. Incluso, desde lo discursivo, las autoridades suecas han realizado algunos cuestionamientos en torno a ciertas características del sistema internacional, y han sostenido disputas diplomáticas con algunos países basadas en cuestiones relacionadas con la vulneración de los Derechos Humanos.

Sin embargo, nada de lo anteriormente mencionado ha sido sostenido a lo largo del tiempo, y las idas y vueltas se han hecho constantes. Notorias discrepancias se han dado en el compromiso con una agenda feminista entre los distintos ministerios, y en algunas áreas, como por ejemplo la defensa, las transformaciones han sido insignificantes. Además, en múltiples ocasiones, Suecia ha abandonado su compromiso con la defensa de postulados feministas, al surgir obstáculos o riesgos para el interés nacional, definido en términos androcentristas. El giro conservador en materia inmigratoria, o la negativa gubernamental a confrontar el estratégico sector de la industria armamentista, son evidencias de ello.

En base al análisis realizado sobre el caso de Canadá, consideramos que este país puede encuadrarse dentro de las PEPG, ya que presenta antecedentes domésticos e internacionales en el trabajo en temáticas referidas a las mujeres. Sin embargo, el hecho de autodefinir su política exterior sólo en un área, como es la asistencia al desarrollo, y enfocar la empírica principalmente en cuestiones económicas, no logra la integralidad necesaria para ubicarla en el nivel superior. Asimismo, Canadá no avanza en críticas ni cuestionamientos, ni tan siquiera discursivos, sobre las dinámicas e instituciones globales hegemónicas, ni problematiza su lugar en la estructura internacional, eso sin mencionar las contradicciones entre su discurso en defensa y las políticas asistencialistas aplicadas.

Por último, evaluando el caso mexicano, nos preguntamos: ¿cómo se puede pensar en una política exterior feminista cuando a nivel doméstico los cárteles del narcotráfico y las redes de tráfico de personas siguen ejerciendo su poder y violencia con total impunidad, y en comunión con amplios sectores de la política, la justicia y la entidad policial? ¿Cómo la PEF mexicana podría 
tener sentido en un país donde las personas siguen "desapareciendo"17 o resultan víctimas de ejecuciones extrajudiciales contra civiles desarmados ${ }^{18}$, en el marco de la democracia, la mayoría de las veces con representantes de las Fuerzas de seguridad como principales responsables? ¿En qué parte del plan de igualdad de género se pueden encuadrar los feminicidios y transfemicidios que colocan a México en el triste podio de uno de los países más peligrosos para ser mujer o persona disidente de la heteronorma? De forma lamentable, consideramos que el país del norte ni siquiera está en condiciones de aspirar al nivel más bajo aquí descrito —es decir, la PEPG-; ya que todas sus acciones actuales (y de las últimas décadas) van en detrimento de los valores y principios que el posicionamiento feminista enarbola.

Las críticas planteadas en los tres casos de estudio abordados, buscaron identificar sus puntos débiles en pos de construir conceptos más adecuados, que señalen los distintos tipos de políticas exteriores que actualmente se orientan hacia las mujeres, y que permitan definirlas con un término apropiado, que se adecúe a su accionar real y no a una mera retórica. Nuestro trabajo, desde luego, no se limita a una corrección conceptual; sino, y sobre todo, intenta generar un aporte concreto para la generación de herramientas y la definición de políticas públicas que impacten de forma positiva en la vida de las comunidades representadas.

\section{Referencias}

Agir Pour la Paix, American Friends Service Committee, Centro de Estudios Ecuménicos, Comisión Mexicana de Defensa y Promoción de los Derechos Humanos, Global Exchange, OPAL, Ohne Rüstung Leben, Nesehnutí y Vredesactie (19.12.2020). Deadly Trade. How European and Israeli arms exports are accelerating violence in Mexico. https://stopusarmstomexico.org/deadly-trade/ (I0.02.202I).

Alwan, C. y Weldon, L. (Junio, 2017).What is Feminist Foreign Policy? An Exploratory Evaluation of Foreign Policy in OECD Countries. Trabajo presentado en la Conferencia Europea sobre Políticas y Género, Lausana.

Amnistía Internacional (22.02.2017). Informe anual 2016/2017. La situación de los Derechos Humanos en el mundo. https://www.amnesty.org/es/documents/poll0/4800/20I7/es/ (I5.07.20I9).

Bäck, H. y Björkdahl, A. (2017). Does Female Leadership Matter? An Analysis of Swedish Foreign Ministers and their Parliamentary Speeches, 1955-20 I6. STANCE Working Papers Series, I0, I-32.

Capar, R.I. (20.10.2020). Tegnell:Larger Immigrant Population Led to Faster Corona Spread in Stockholm. https://norwaytoday. info/news/tegnell-larger-immigrant-population-led-to-faster-corona-spread-in-stockholm/ (05.I I.2020).

CEAR - Comisión Española de Ayuda al Refugiado (2019). Retos y oportunidades del sistema de asilo en Suecia. https:// www.cear.es/wp-content/uploads/2019/04/INFORME-FINAL-SUECIA.pdf (I8.05.2020).

CEPAL - Comisión Económica para América Latina y el Caribe (2010).“¿Qué Estado para qué igualdad?” Declaración de las mujeres indígenas y afrodescendientes de América Latina, el Caribe y la diáspora. En Espinosa Miñoso, Y., Gómez Correal, D. y Ochoa Muñoz, K. (Eds.) (20l4). Tejiendo de otro modo: Feminismo, epistemología y apuestas descoloniales en Abya Yala (pp. 435-437). Editorial Universidad del Cauca.

Committee to Protect Journalists (2020). 9 Journalists Killed in Mexico. https://cpj.org/data/killed/202I/?status=Killed\& motiveConfirmed\%5B\%5D=Confirmed\&motiveUnconfirmed\%5B\%5D=Unconfirmed\&type\%5B\%5D=Journ alist\&cc_fips\%5B\%5D=MX\&start_year=2020\&end_year=2020\&group_by=location (28.02.202I).

Consejo de Seguridad de Naciones Unidas (202I) Funciones y poderes. https://www.un.org/securitycouncil/es/content/

17 El concepto de "desaparecidos/as" fue muy difundido en Sudamérica, durante los gobiernos dictatoriales. Estados genocidas que secuestraron, torturaron y asesinaron a quienes tenían el deber de proteger, en tanto ciudadanos/as de sus territorios. Aunque desde el regreso a la democracia pueden mencionarse algunos casos de "desaparición", por ejemplo, el caso de Julio López y María Cash en Argentina, afortunadamente no puede decirse que es habitual; como sí podemos evidenciar en el caso mexicano. De acuerdo a un informe conjunto, realizado por Global Exchange,Vredesactie, OPAL, Agir pour la Paix, American Friends Service Committee, Ohne Rüstung Leben, NESEHNUTÍ, la Comisión Mexicana de Defensa y Promoción de los Derechos Humanos, y el Centro de Estudios Ecuménicos, unas "73 mil personas están registradas como desaparecidas en México, más de 56.000 desaparecidas en la última década. A noviembre de 2020, quedan más de 37.000 cadáveres no identificados" (2020, p. 3, traducción propia).

18 Tal como sostiene Estéves López (2015, p. 7): "Las masacres recientes de Apatzingán, Ayotzinapa y Ecuandureo, en las que se ha comprobado la participación de las fuerzas del Estado, al igual que de criminales, indican no sólo un grado de corrupción y descomposición político-administrativa de proporciones gigantescas, sino que la administración de la muerte para el control del capital criminal es sistemática y cada vez más generalizada”. 
functions-and-powers (25.02.202I).

Curiel Pichardo, O. (I0.08.202I). Los feminismos críticos. Análisis complejos de la matriz de opresión”. Red de Trabajadoras por la Educación, Costa Rica. https://www.facebook.com/watch/live/?v=882 | 46 |45727487\&ref =watch_permalink (I4.08.202I).

Dahl, R. (2004). La democracia. Revista POSTData, I0, I I-55.

Data Cívica, Equis Justicia para las Mujeres, e Intersecta Organización para la lgualdad (diciembre 2020). Falsas salvaguardas. Las capacitaciones de las Fuerzas Armadas en Derechos Humanos y Género (2010-2019). https://datacivica.org/assets/pdf/informe falsas salvaguardas.pdf (27.02.202I).

Delgado Peralta, M. (2020). La política exterior feminista de México y la agenda de la sostenibilidad 2030. Revista Mexicana de Política Exterior, I 18, |3।-138.

Gargallo, F. (2009). Feminismo y globalización:Una mirada desde América Latina. En Berlanga, M., Ferreyra,J.L., Gargallo, F., Mogrovejo, N. y Nuño, S.E. Mujer y violencia: el feminismo en la era de la globalización (pp. 87- I02). Universidad Autónoma de la Ciudad de México.

Gargallo, F. (2015). Feminismos desde Abya Yala: Ideas y proposiciones de las mujeres de 607 pueblos en Nuestra América. Universidad Autónoma de la Ciudad de México.

Global Affairs Canada (2016). Exports of military goods 20/6. https://www.international.gc.ca/controls-controles/ report-rapports/mil-2016.aspx?lang=eng (I2.02.202I).

Global Affairs Canada (2017a). Exports of military goods 20I7. https://www.international.gc.ca/controls-controles/ report-rapports/mil-20 I 7.aspx?lang=eng (I 2.02.202I).

Global Affairs Canada (20I7b). Canada's Feminist International Assistance Policy. https://www.international.gc.ca/worldmonde/issues_development-enjeux_developpement/priorities-priorites/policy-politique.aspx?lang=eng (| 2.02.202|).

Global Affairs Canada (2018). Exports of military goods 2018. https://www.international.gc.ca/trade-commerce/controlscontroles/reports-rapports/military_goods-2018-marchandises_militaires.aspx?lang=eng (I 2.02.202I).

Global Affairs Canada (2019). Exports of military goods 2019. https://www.international.gc.ca/trade-commerce/controlscontroles/reports-rapports/military-goods-20I9-marchandises-militaries.aspx?lang=eng (I 2.02.202I).

Goertz, G. y Mazur, A.G. (2008). Mapping Gender and Politics Concepts:Ten Guidelines. En Goertz. G. y Mazur, A.G. (Ed.) Politics, Gender and concepts. Theory and methodology (pp. 14-43). Cambridge University Press.

Gómez Grijalva, D. (20I3). Mi cuerpo es un territorio político. Voces Descolonizadoras, Cuaderno I. Brecha Lésbica.

Georgetown Institute for Women, Peace and Security y The Peace Research Institute Oslo (2019). Women, Peace, and Security Index 2019/20. https://giwps.georgetown.edu/wp-content/uploads/2019/I2/WPS-Index-20I920-Report.pdf (26.02.202I).

Lugones, M. (2008). Colonialidad y género. Tabula rasa, 9, 73-I0I.

Lugones, M. (202I). Peregrinajes:Teorizar una coalición contra múltiples opresiones. Del Signo.

Lunz, K. y Bernarding, N. (13.02.20I9). Feminist Foreign Policy. Imperative for a More Secure and Just World. En Feministische Außenpolitik (s/d). Heinrich-Böll-Stiftung.

Mendoza, B. (20l0). La epistemología del sur, la colonialidad del género y el feminismo latinoamericano. En Espinosa Miñoso, Y. (Coord.). Aproximaciones críticas a las prácticas teórico-políticas del feminismo latinoamericano (pp. 19 36). En La Frontera.

Mesa, M. (202I). Política exterior feminista: la apuesta de los gobiernos por la igualdad. Anuario CEIPAZ 2020-202I, $13,113-142$.

Mignolo, W. (2003). Historias locales / diseños globales. Colonialidad, conocimientos subalternos y pensamiento fronterizo. Akal.

Ministerio de Asuntos Exteriores de Suecia (23.08.20I8). Manual de Política Exterior Feminista. https://www.government. se/reports/2018/08/handbook-swedens-feminist-foreign-policy/ (20.04.202I).

Ministerio de Asuntos Exteriores de Suecia (2019). Manual Política exterior feminista de Suecia. https://www.google. $\mathrm{com} / \mathrm{url}$ ?sa=t\&rct=i\&q=\&esrc=s\&source=web\&cd=\&cad=rja\&uact=8\&ved=2ahUKEwiW7a3Ex4jI AhXyRPE DHfPuBoQQFnoECAIQAQ\&url=https\%3A\%2F\%2Fwww.government.se\%2F492c36\%2Fcontentassets\%2Ff c l I 5607a4ad4bca9 I 3cd8d I I c2339dc\%2Fhandbook---swedens-feminist-foreign-policy---spanish.pdf\&usg=A OvVawIMtGiS57ffuOrLhCqcLMhn

Ministerio de Justicia de Suecia (03.05.2018) Internal border controls prolonged. https://www.government.se/pressreleases/2018/05/internal-border-controls-prolonged/ (22.10.2020).

Morton, S.; Muchiri, J. y Swiss, L. (2020). Which Feminism(s)? For Whom? Intersecctionality in Canada's Feminist International Assistance Policy. International Journal, 75 (3), 329-348.

Naciones Unidas (20l8). Compendio de implementación modular de control de armas pequeñas. Mujeres, hombres y la naturaleza de género de las armas pequeñas y ligeras. https://iansa.org/wp-content/uploads/202I/04/MOSAIC06. I0-20I7SVI.0.pdf (I5.03.202I)

Oficina del Primer Ministro de Suecia (24.I I.20I5). Government proposes measures to create respite for Swedish refugee reception. https://www.government.se/articles/20I5/I I/government-proposes-measures-to-create-respitefor-swedish-refugee-reception/ (I I.02.202I).

Parisi, L. (2020). Canada's New Feminist International Assistance Policy: Business as Usual? Foreign Policy Analysis, 0 , I- 18.

Quijano, A. (1992). Colonialidad y modernidad/racionalidad. Perú indígena, I3, I I-20. 
Richard, N. (2013). Multiplicar la(s) diferencia(s): género, política, representación y deconstrucción. En Grimson, A. (Coord.) Hegemonía cultural y política de la diferencia (pp. I35-I46). CLACSO.

Secretaría de Defensa Nacional de México (2020). Acciones y programas.

https://www.gob.mx/sedena/acciones-y-programas/corte-interamericana-de-derechos-humanos-caso-no-12-916 (I0.02.202I)

SIPRI (2019). Trends in international arms transfers, 20/8. https://www.sipri.org/publications/2019/sipri-fact-sheets/ trends-international-arms-transfers-20I8 (I6.02.202I).

SIPRI (09.03.2020a). Trends in international arms transfers, 2019. https://www.sipri.org/publications/2020/sipri-factsheets/trends-international-arms-transfers-2019 (I6.02.202I).

SIPRI (26.04.2020b). Trends in World Military Expenditure, 2019. https://www.sipri.org/publications/202I/sipri-factsheets/trends-world-military-expenditure-2020\#: :text=World\%20military\%20expenditure\%20in\%20 2020\%20was\%202.6\%20per\%20cent\%20higher,2020\%2C\%20to\%202.4\%20per\%20cent. (I6.02.202I).

Smith, H. y Ajadi, T. (2020). Canada's Feminist Foreign Policy and Human Security Compared. International Journal, 75 (3), 367-382.

Spivak, G. (1990). Criticism, Feminism and the Institutio. En Spivak, G. The Post-Colonial:Interviews, Strategies, Dialogues (pp. I35- I46). Routledge.

Stutzin,V.y Troncoso, L. (2019). La agenda heteropatriarcal en Chile: Cruces entre política, moral y religión en la lucha contra la "ideología de género". Nomadías, 28, 9-4I.

Svampa, M. y Slipak, A. (20I5). China en América Latina: Del Consenso de los commodities al Consenso de Beijing. Ensambles, 3, 34-63.

Thompson, L. and Clement, R. (2019). Defining Feminist Foreign Policy. International Center for Research on Women.

US.News \& World Report (2019). México Rankings. https://www.usnews.com/news/best-countries/mexico\#countryranking-detail (26.02.202I).

Velázquez, K. (30.03.2021). La industria de la chatarra se chupa el agua de comunidades y las deja secas, acusan expertos. https://www.sinembargo.mx/30-03-202 I/3956746

Viveros Vigoya, M. (20I6). La interseccionalidad: una aproximación situada a la dominación. Debate Feminista, 52 , I-I 7. 


\section{RELACIONES INTERNACIONALES}

Revista académica cuatrimestral de publicación electrónica Grupo de Estudios de Relaciones Internacionales (GERI)

Universidad Autónoma de Madrid, España

https://revistas.uam.es/relacionesinternacionales

ISSN 1699 - 3950

f facebook.com/RelacionesInternacionales

3. twitter.com/RRInternacional

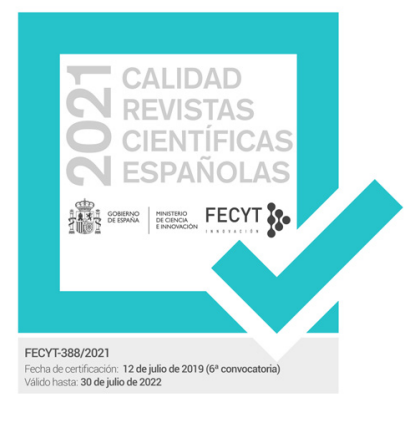

